\title{
Surgical resection and long-term disease-free survival in stage IIIB non-small cell lung cancer after gefitinib down- staging: a case report
}

\author{
Natella Jafarova ${ }^{1}$, Gary Dewar ${ }^{2}$, R Petter Tonseth ${ }^{3}$ and David L. Saltman ${ }^{* 1}$ \\ ${ }^{1}$ Department Medical Oncology, British Columbia Cancer Agency, 2410 Lee Avenue, Victoria, British Columbia, Canada \\ ${ }^{2}$ Department of Thoracic Surgery, Royal Jubilee Hospital, 1952 Bay Street, Victoria, British Columbia, Canada \\ ${ }^{3}$ Department of Functional Imaging, BC Cancer Agency, Vancouver, British Columbia, Canada
}

\begin{abstract}
The use of tyrosine kinase inhibitors to treat advanced stage non-small cell lung cancer tumors harboring epidermal growth factor receptor (EGFR) activating mutations can result in improved response rates and progression-free survival compared with cytotoxic chemotherapy. However, most patients will have progression of their cancer within 8 to 12 months. Down-staging of inoperable disease after tyrosine kinase inhibitor therapy may permit an attempt at resection of the primary tumor. However, most patients' disease will progress after surgery even with the use of adjuvant tyrosine kinase therapy. We report the case of a patient with cT1N3M0 non-small cell lung cancer harboring an epidermal growth factor receptor activating mutation. Radiologic down-staging to N0 disease with gefitinib allowed surgical resection of the primary lung tumor 4 years after initiating TKI therapy. The patient received adjuvant gefitinib for 8 months following her surgery, but then discontinued the drug. Twenty-two months after surgery and 11 months after discontinuing gefitinib there is no evidence of recurrent cancer. Lung cancer patients with prolonged eradication of metastatic disease while receiving tyrosine kinase inhibitors may benefit from surgical resection of their primary tumors.
\end{abstract}

\begin{abstract}
Abbreviations: NSCLC: non-small cell lung cancer; SABR: stereotactic ablative radiotherapy; EGFR: epidermal growth factor receptor; TKI: tyrosine kinase inhibitor; CT: computed tomography; FDG-PET: fluorodeoxyglucose-positron emission tomography; DFS: disease-free survival
\end{abstract}

\section{Introduction}

Lung cancer remains the leading cause of cancer related deaths [1]. Unfortunately, the majority of patients will present with advanced stage disease [1]. In the case of non-small cell lung cancer (NSCLC), there is evidence to support the use of neoadjuvant combined modality radiation and chemotherapy in stage IIIA disease [2]. Although there may be benefit for neoadjuvant therapy followed by surgery in selected cases of cT4N2 stage IIIB disease, cTxN3 cases are excluded from this approach and treated the same as stage IV disease with palliative chemotherapy and radiation [2].

The use of epidermal growth factor receptor (EGFR) tyrosine kinase inhibitors (TKIs) for EGFR activating mutation-positive advanced stage NSCLC has resulted in higher response rates and progression-free survivals when compared to cytotoxic chemotherapy, but the majority of patients will progress on treatment within one year [3,4]. Aggressive treatment of oligoprogression with focal therapies, such as stereotactic ablative radiotherapy (SABR), while continuing to use tyrosine kinase inhibitors can result in prolonged survivals, but few patients will be cured of their disease [5]. Here we present a case of biopsy proven cT1N3M0 EGFR mutation-positive NSCLC treated with an EGFR inhibitor resulting in radiological down-staging. Oligoprogression in the primary tumor was treated by surgical resection, followed by discontinuation of the EGFR inhibitor without evidence of recurrence two years after surgery.

\section{Case presentation}

A 78 year-old Caucasian female never-smoker with no prior history of cancer presented in 2009 with a nonproductive cough. The patient had a history of hypertension and supraventricular arrhythmias, both managed with medication. A computed tomography (CT) scan demonstrated a $1.5 \mathrm{~cm}$ spiculated nodule in the posteromedial segment of right lower lobe of the lung. A bronchoscopic biopsy confirmed the suspicion of non-small cell lung cancer, adenocarcinoma. A fluorodeoxyglucose-positron emission tomography (FDG-PET) scan revealed FDG-avidity in a $2 \mathrm{~cm}$ nodule in the right lower lobe and a subcarinal lymph node (Figures 1A and 1B). Mediastinoscopy was performed and demonstrated metastatic involvement of a sternal notch lymph node. CT scan of the brain was negative for metastatic disease. The final staging was cT1aN3M0 (IIIB). The patient was offered chemotherapy but declined in favor of close observation. EGFR mutational analysis was performed four months later and was positive for an exon 19 deletion. The patient was started on gefitinib $250 \mathrm{mg}$ daily and responded well with a marked decrease in the size

Correspondence to: David L. Saltman, Department Medical Oncology, British Columbia Cancer Agency, 2410 Lee Avenue, Victoria, British Columbia, Canada, E-mail: David.saltman@bccancer.bc.ca

Key words: epidermal growth factor receptor, gefitinib, neoadjuvant, non-small cell lung cancer, tyrosine kinase inhibitor, salvage surgery

Received: February 24, 2016; Accepted: March 04, 2016; Published: March 08 2016 
A

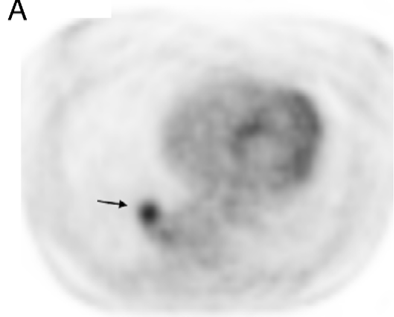

C

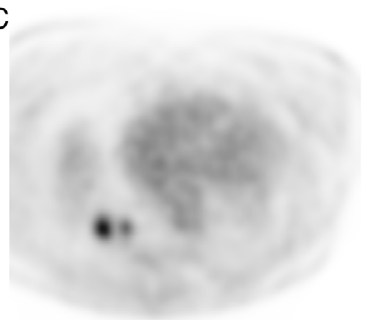

$\mathrm{E}$

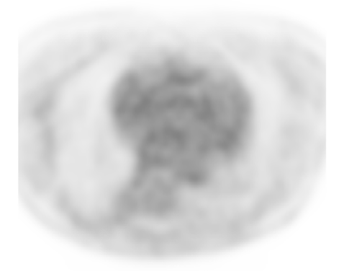

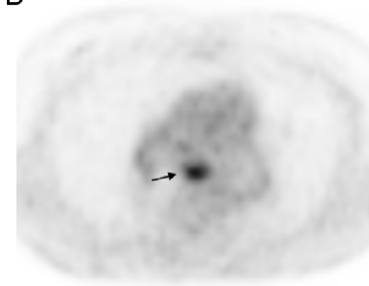

D

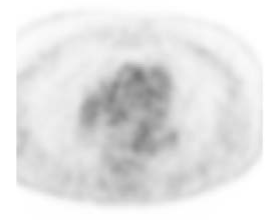

$\mathrm{F}$

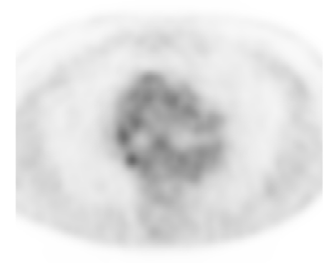

Figure 1. Representative chest FDG-PET images at diagnosis pre-gefitinib, and before and after surgery. A and B; FDG-avid primary tumor in the right lower lobe and subcarinal lymph node. C and D; Persistent FDG-avidity in the primary tumor with complete response in the subcarinal lymph node. E and F; FDG-PET four months after surgery demonstrating no evidence of FDG-avid disease.
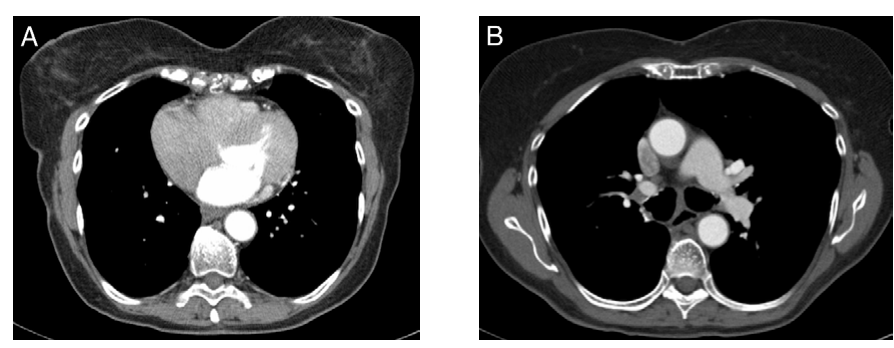

Figure 2. CT-scan images of the chest 22 months after surgery and 11 months after discontinuing gefitinib demonstrating no evidence of recurrent disease in the right lung (A) or the mediastinum (B).

of the primary lesion from 3.5 to $2.1 \mathrm{~cm}$ after six weeks on treatment. The $1.6 \mathrm{~cm}$ subcarinal node noted at the start of gefitinib was no longer enlarged. The dose of the gefitinib was later changed to $250 \mathrm{mg}$ every day except Sunday due to diarrhea. The patient continued on close surveillance and had no radiological evidence of disease progression until 3.5 years from the start of gefitinib when a slight increase in the size of the lung lesion was noted. The patient continued to take gefitinib with further slow progression even on full doses. A FDG-PET scan 5 months later confirmed the progression of the disease in the right lower lobe but no evidence of disease in the mediastinum or outside the chest (Figures 1C and 1D). The patient was offered a choice of radiation to the RLL mass or surgical resection, and the latter was selected. A right lower lobectomy was performed and tolerated well by the patient. The pathology showed a $3 \mathrm{~cm}$ focally necrotic mass with visceral pleural involvement, but negative resection margins. The tumor was a poorly

differentiated adenocarcinoma. One of nine lymph nodes was involved. The final pathologic staging was ypT2aN1M0. Six weeks after the surgery, the patient restarted gefitinib but at a reduced dose of $250 \mathrm{mg}$ every other day, due to concerns over side effects. Four months after surgery a FDG-PET scan showed no active disease (Figures $1 \mathrm{E}$ and $1 F)$. She subsequently decided to discontinue using the gefitinib, after 8 months of adjuvant use, because of concerns regarding side effects including diarrhea and fatigue. A CT scan performed 22 months after surgery and 11 months after discontinuing gefitinib shows no evidence of disease (Figure 2).

\section{Discussion}

Despite the convincing data to support the use of tyrosine kinase inhibitors in advanced NSCLC with activating mutations in EGFR, there is limited evidence to date to support the use of these agents in the neoadjuvant setting in early stage disease. Evidence has largely come from case reports and a small number of Phase II trials that focused mostly on response rates rather than survival $[6,7]$.

The results of studies of adjuvant EGFR TKI therapy after chemoradiation or surgery for early stage NSCLC have also been inconclusive. Retrospective and prospective studies of adjuvant TKIs after surgical resections of stage I to IIIA disease have yielded conflicting outcomes. The negative results of the Canadian NCIC CTG BR19 study were difficult to interpret because patients were not selected on the basis of there EGFR mutational status [8]. There was an improvement in disease-free survival (DFS) in a more recent randomized, phase II trial of gefitinib after adjuvant chemotherapy in resected stage IIIA NSCLC [9]. A phase II study using erlotinib in resected early stage NSCLC with activating EGFR mutations demonstrated an improvement in DFS but the authors used a historical control group for comparison [10]. In a phase III study of stage IIA-IIIA NSCLC patients randomized to erlotinib versus placebo after surgical resection plus or minus adjuvant chemotherapy there was no statistical difference between the two study groups in the subset of patients who were EGFR mutation-positive [11].

There is limited evidence to support surgical resection of primary and oligometastatic lesions in more advanced stage inoperable NSCLC after treatment with EGFR TKIs [12-19]. Complete pathological responses have been reported after neoadjuvant use of EGFR TKIs and salvage surgery, but these are uncommon. In a report of nine patients with stage IIIA-IV disease only one patient with cT2N2M0 at diagnosis and three years of neoadjuvant gefitinib had a complete pathologic response after salvage surgery [12]. The patient did not receive adjuvant gefitinib, and developed brain metastases 28 months after surgery. The remaining group of patients, who had stage IIIB and IV at diagnosis, all had residual disease at surgery and a short medial progression-freesurvival of only 6 months. There was no survival advantage associated with adjuvant gefitinib in this series but the number of patients reported was small. Weber, et al. (2013) reported two cases of advanced lung cancer with activating mutations in EGFR (deletion exon 19), who underwent surgical resection of the lung primaries after treatment with erlotinib. Both patients had complete pathologic responses in their surgical specimens. At the time of publication, both patients had long disease-free intervals since surgery. One of the two patients discontinued the TKI after surgery but only after a lengthy interval of 4 years. These cases are in contrast to our patient who had a residual tumor in the primary lung lesions and one lymph node at the time of salvage surgery and only received adjuvant gefitinib for 8 months, but 
is still disease-free close to 2 years after surgery.

\section{Conclusions}

Tyrosine kinase inhibitor therapy for the treatment of advanced stage NSCLC with activating mutations in EGFR may result in sustained radiologic down-staging, allowing for surgical resection of primary lung tumors that are deemed unresectable at diagnosis. Removal of the primary tumor in this setting may help to prevent the dissemination of TKI resistant tumor cells to more distant sites. This may result in an improvement in survival, and in some patients allow for the discontinuation of TKIs without evidence of recurrent disease after long-term follow-up.

\section{Consent}

Written informed consent was obtained from the patient for publication of this case report and any accompanying images. A copy of the written consent is available for review from the Editor of this journal.

\section{Competing interests}

The authors declare no competing interests.

\section{Authors contributions}

NJ reviewed the clinical data, drafted and critically reviewed the manuscript. RPT reviewed the imaging studies and help draft and review the manuscript. GD managed the surgical care of the patient, reviewed the data and reviewed the manuscript. DS treated the patient, reviewed the data and drafted and critically reviewed the manuscript. All authors read and approved the final manuscript.

\section{References}

1. Siegel RL, Miller KD, Jemal A (2015) Cancer statistics, 2015. CA Cancer J Clin 65: 5-29. [Crossref]

2. Eberhardt WEE, De Ruysscher D, Weder W, Le Péchoux C, De Leyn P, et al. (2015) $2^{\text {nd }}$ ESMO consensus conference in lung cancer: locally advanced stage III non-small-cell lung cancer. Ann Oncol 26:1573-1588. [Crossref]

3. Mok TS, Wu YL, Thongprasert S, Yang CH, Chu DT, et al. (2009) Gefitinib or carboplatin-paclitaxel in pulmonary adenocarcinoma. N Engl J Med 361:947-957. [Crossref]

4. Lee CK, Brown C, Gralla RJ, Hirsh V, Thongprasert S, et al. (2013) Impact of EGFR inhibitor in non-small cell lung cancer on progression-free and overall survival: a metaanalysis. J Natl Cancer Inst 105: 595-605. [Crossref]

5. Weickhardt AJ, Scheier B, Burke JM, Gan G, Lu X, et al. (2012) Local ablative therapy of oligoprogressive disease prolongs disease control by tyrosine kinase inhibitors in oncogene-addicted non-small-cell lung cancer. J Thorac Oncol 7: 1807-1814. [Crossref]
6. Lara-Guerra H, Waddell TK, Salvarrey MA, Joshua AM, Chung CT, et al. (2009) Phase II study of preoperative gefitinib in clinical stage I non-small-cell lung cancer. $J$ Clin Oncol 27: 6229-6236. [Crossref]

7. Schaake EE, Kappers I, Codrington HE, Valdés Olmos RE, Teerstra HJ, et al. (2012) Tumor response and toxicity of neoadjuvant erlotinib in patients with early-stage nonsmall cell lung cancer. J Clin Oncol 30:2731-2738. [Crossref]

8. Goss GD, O'Callaghan C, Lorimer I (2013) Gefitinib versus placebo on completely resected non-small cell lung cancer: results of the NCIC CTG BR19 study. J Clin Oncol 31: $3320-3326$.

9. LI N, Ou W, Ye X, Sun HB, Zhang L, et al. (2014) Premetrexed-carboplatin adjuvant chemotherapy with or without gefitinib in resected stage IIIA-N2 non-small cell lung cancer habouring EGFR mutations: a randomized, phase II study. Ann Surg Oncol 21: 2091-2096. [Crossref]

10. Pennell NA, Neal JW, Chaft JE, et al. (2014) SELECT: A multicenter phase II trial of adjuvant erlotinib in resected early-stage EGFR mutation-positive NSCLC. $J$ Clin Oncol 32 (15 suppl; abstr 7514).

11. Kelly K, Altorki NK, Eberhardt WE, O'Brien ME, Spigel DR, et al. (2015) Adjuvan erlotinib versus placebo following complete tumor resection with stage IB-IIIA nonsmall cell lung cancer. RADIANT: A randomized, double-blinded placebo trial. J Clin Oncol 33: 4007-4014.

12. Takamochi K, Suzuki K, Sugimura H, Funai K, Mori H, et al. (2007) Surgical resection after gefitinib treatment in patients with lung adenocarcinoma harboring epidermal growth factor receptor gene mutation. Lung Cancer 58: 149-155. [Crossref]

13. Levchenko EV, Moiseyenko VM, Matsko DE, Iyevleva AG, Ivantsov AO, et al. (2009) Down-staging of EGFR mutation-positive advanced lung carcinoma with gefitinib followed by surgical intervention: follow-up of two cases. Onkologie 32:674-677. [Crossref]

14. Hishida T, Nagai K, Mitsudomi T, Yokoi K, Kondo H, et al. (2010) Salvage surgery for advanced non-small cell lung cancer after response to gefitinib. $J$ Thorac Cadiovasc Surg 140:e69-71. [Crossref]

15. Ayabe T, Shimizu T, Tomita M, Hara M, Onitsuka T (2011) A case of long-term survival of gefitinib-induction therapy for advanced N2-multistation lung cancer without epidermal growth factor receptor gene mutation. Ann Cancer Res Therap 19: 62-65.

16. Liu M, Jiang G, He W, Zhang P, Song N (2011) Surgical resection of locally advanced pulmonary adenocarcinoma after gefitinib therapy. Ann Thorac Surg 92: e11-12. [Crossref]

17. Ong M, Kwan K, Kamel-Reid S, Vincent M (2012) Neoadjuvant erlotinib and surgical resection of a stage IIIA papillary adenocarcinoma of the lung with an L861Q activating EGFR mutation. Curr Oncol 19: e222-226. [Crossref]

18. Weber B, Sorensen BS, Knap MM, Madsen HH, Nexo E, et al. (2011) Complete pathologic response in lung tumors in two patients with metastatic non-small cell lung cancer treated with erlotinib. J Thorac Oncol 6: 1946-1949. [Crossref]

19. López-González A, Almagro, E, Salas C, Varela A, Provencio M (2013) Use of tyrosine kinase inhibitor as neoadjuvant therapy for non-small cell lung cancer: A case report. Resp Med Case Rep 9: 8-10. [Crossref]

Copyright: (C2016 Jafarova N. This is an open-access article distributed under the terms of the Creative Commons Attribution License, which permits unrestricted use, distribution, and reproduction in any medium, provided the original author and source are credited. 